\title{
Stern polynomials and double-limit continued fractions
}

by

Karl Dilcher (Halifax) and Kenneth B. Stolarsky (Urbana, IL)

1. Introduction. One of the most remarkable integer sequences in number theory and combinatorics is the Stern sequence, sometimes also called Stern's diatomic sequence. This sequence $\{a(n)\}_{n \geq 0}$ is given by $a(0)=0$, $a(1)=1$, and for $n \geq 1$ by

$$
a(2 n)=a(n), \quad a(2 n+1)=a(n)+a(n-1) .
$$

For numerous properties and references, see, e.g., [24, A002487] or [23].

Recently the authors [13] defined a polynomial analogue as follows. Let $a(0 ; x)=0, a(1 ; x)=1$, and for $n \geq 1$ let

$$
\begin{aligned}
a(2 n ; x) & =a\left(n ; x^{2}\right), \\
a(2 n+1 ; x) & =x a\left(n ; x^{2}\right)+a\left(n+1 ; x^{2}\right) .
\end{aligned}
$$

We call the polynomial $a(n ; x)$ the $n$th Stern polynomial. Numerous properties of these polynomials can be found in [13]; here we only repeat the obvious properties

$$
a(n ; 0)=1 \quad(n \geq 1), \quad a(n ; 1)=a(n) \quad(n \geq 0),
$$

and, for all $m \geq 0$,

$$
a\left(2^{m} ; x\right)=1
$$

this last identity follows by iterating (1.2). For ease of reference we also list the first Stern polynomials up to $n=32$ in Table 1.

We note that a different polynomial extension of the Stern sequence (1.1), also called Stern polynomials, was introduced and studied in [16].

An important and interesting property of the Stern sequence is the fact that in each interval $2^{n-2} \leq m \leq 2^{n-1}$ the maximum value of $a(m)$ is the Fibonacci number $F_{n}$. In general this maximum is attained twice, at well-known and specified indices $m$; we let $\alpha_{n}$ and $\beta_{n}$ be these indices in

2010 Mathematics Subject Classification: Primary 11B37; Secondary 11B75, 11B83, 11J70. Key words and phrases: Stern sequence, recurrence relations, polynomial sequences, functional equations, continued fractions. 
Table 1. $a(n ; x), 1 \leq n \leq 32$

\begin{tabular}{rlll}
\hline \multicolumn{1}{c}{$a(n ; x)$} & $n$ & \multicolumn{1}{c}{$a(n ; x)$} \\
\hline 1 & 1 & 17 & $1+x+x^{2}+x^{4}+x^{8}$ \\
2 & 1 & 18 & $1+x^{2}+x^{4}+x^{8}$ \\
3 & $1+x$ & 19 & $1+x+x^{3}+x^{4}+x^{5}+x^{8}+x^{9}$ \\
4 & 1 & 20 & $1+x^{4}+x^{8}$ \\
5 & $1+x+x^{2}$ & 21 & $1+x+x^{2}+x^{5}+x^{6}+x^{8}+x^{9}+x^{10}$ \\
6 & $1+x^{2}$ & 22 & $1+x^{2}+x^{6}+x^{8}+x^{10}$ \\
7 & $1+x+x^{3}$ & 23 & $1+x+x^{3}+x^{7}+x^{8}+x^{9}+x^{11}$ \\
8 & 1 & 24 & $1+x^{8}$ \\
9 & $1+x+x^{2}+x^{4}$ & 25 & $1+x+x^{2}+x^{4}+x^{9}+x^{10}+x^{12}$ \\
10 & $1+x^{2}+x^{4}$ & 26 & $1+x^{2}+x^{4}+x^{10}+x^{12}$ \\
11 & $1+x+x^{3}+x^{4}+x^{5}$ & 27 & $1+x+x^{3}+x^{4}+x^{5}+x^{11}+x^{12}+x^{13}$ \\
12 & $1+x^{4}$ & 28 & $1+x^{4}+x^{12}$ \\
13 & $1+x+x^{2}+x^{5}+x^{6}$ & 29 & $1+x+x^{2}+x^{5}+x^{6}+x^{13}+x^{14}$ \\
14 & $1+x^{2}+x^{6}$ & 30 & $1+x^{2}+x^{6}+x^{14}$ \\
15 & $1+x+x^{3}+x^{7}$ & 31 & $1+x+x^{3}+x^{7}+x^{15}$ \\
16 & 1 & 32 & 1 \\
\hline
\end{tabular}

the interval. The main object of study in this paper is the subsequence of Stern polynomials with index $\alpha_{n}$, along with a companion sequence of polynomials with index $\beta_{n}$. These polynomials will be introduced and studied in Section 3. Because of their close relationship to the Fibonacci numbers, they can be considered either as polynomial analogues or, to some extent, also as $q$-analogues of Fibonacci numbers. We therefore chose the letter $q$ as variable.

Before we define these polynomials in Section 3, we prove a crucial result on the Stern polynomials which goes beyond [13] and is the basis of much of what follows in this paper; this will be done in Section 2. In Section 4 we derive several quadratic identities, and in Section 5 we define and investigate two analytic functions as limits of the above polynomial sequences. Section 6 then deals with some finite and infinite continued fractions. In a case of particular interest, the set of convergents has exactly two limit points. Finally, in Section 7, we prove some transcendence results for the infinite continued fractions obtained in the previous section.

2. A result on Stern polynomials. In order to motivate the main result in this section, we consider patterns in the polynomials $a(n ; x)$ that are somewhat less obvious than those induced by (1.2) or (1.3). For instance, in $a(27 ; x)$ (see Table 1) the two-fold occurrence of three consecutive powers 
of $x$ indicates that we can write

$$
\begin{aligned}
a(27 ; x) & =x^{3}\left(1+x+x^{2}\right)\left(1+x^{8}\right)+(1+x) \\
& =x^{3} a(5 ; x) a\left(3 ; x^{8}\right)+a(3 ; x) .
\end{aligned}
$$

This is a special case of the following result.

Proposition 2.1. Let $j, m, n$ be integers with $0 \leq m \leq n$ and $0 \leq j$ $\leq 2^{m}$. Then

$$
a\left(2^{n}-j ; x\right)=x^{2^{m}-j} a(j ; x) a\left(2^{n-m}-1 ; x^{2^{m}}\right)+a\left(2^{m}-j ; x\right) .
$$

Before proving this result we derive a few consequences. First, with $n=5, m=3$, and $j=5$ we immediately get our example (2.1). Furthermore, if we set $x=1$ then with (1.4) we get the following result on the Stern sequence which we have not been able to find in the literature.

Corollary 2.1. Let $j, m, n$ be integers with $0 \leq m \leq n$ and $0 \leq j \leq 2^{m}$. Then

$$
a\left(2^{n}-j\right)=a(j) a\left(2^{n-m}-1\right)+a\left(2^{m}-j\right) .
$$

The next corollary is equivalent to Lemma 2.1 in [13], but is presented here in a different form:

Corollary 2.2. For integers $m \geq 0$ and $0 \leq j \leq 2^{m}$ we have

$$
x^{j} a\left(2^{m+1}-j ; x\right)=\left(x^{2^{m}}-1\right) a(j ; x)+a\left(2^{m}+j ; x\right) .
$$

Proof. First we set $n=m+1$ in (2.2) and replace $2^{m}-j$ by $j$. This gives

$$
a\left(2^{m}+j ; x\right)=x^{j} a\left(2^{m}-j ; x\right)+a(j ; x),
$$

which is just Lemma 2.1 in [13]. Next we set again $n=m+1$ in (2.2) and multiply both sides by $x^{j}$, to obtain

$$
x^{j} a\left(2^{m+1}-j ; x\right)=x^{2^{m}} a(j ; x)+x^{j} a\left(2^{m}-j ; x\right) .
$$

Now we combine this with (2.5) and immediately obtain (2.4).

If we set $x=1$ in (2.4) we obtain the well-known reflection property for the Stern sequence, namely

$$
a\left(2^{m+1}-j\right)=a\left(2^{m}+j\right)
$$

for $m \geq 0$ and $0 \leq j \leq 2^{m}$. The identity (2.4) can therefore be seen as a polynomial analogue of this reflection property.

Proof of Proposition 2.1. We begin with the case $m=0$; then $j=0$ or 1 , and (2.2) becomes

$$
a\left(2^{n}-j ; x\right)=x^{1-j} a(j ; x) a\left(2^{n}-1 ; x\right)+a(1-j ; x) .
$$

Both cases $(j=0$ or 1$)$ are obvious if we use the fact that $a(0 ; x)=0$ and $a(1 ; x)=1$ hold identically for all $x$. 
The main proof is now by induction on $n$. When $n=0$, then $m=0$ and $j=0$ or 1 , so the start of the induction is a special case of (2.6). For the induction step we assume that (2.2) holds up to some $n-1$. First, if $j=2 k$ is even, we use (1.2) and the induction hypothesis with $0 \leq m \leq n-1$ and $x^{2}$ in place of $x$ to obtain

$$
\begin{aligned}
a\left(2^{n}-2 k ; x\right) & =a\left(2^{n-1}-k ; x^{2}\right) \\
& =\left(x^{2}\right)^{2^{m}-k} a\left(k ; x^{2}\right) a\left(2^{n-1-m}-1 ;\left(x^{2}\right)^{2^{m}}\right)+a\left(2^{m}-k ; x^{2}\right) \\
& =x^{2^{m+1}-2 k} a(2 k ; x) a\left(2^{n-(m+1)}-1 ; x^{2^{m+1}}\right)+a\left(2^{m+1}-2 k ; x\right) .
\end{aligned}
$$

Second, when $j=2 k+1$ is odd, we write $2^{n}-j=2\left(2^{n-1}-k-1\right)+1$ and use (1.3) and the induction hypothesis, once again with $0 \leq m \leq n-1$ and $x^{2}$ in place of $x$ :

$$
\begin{aligned}
a\left(2^{n}-\right. & 2 k-1 ; x) \\
= & x a\left(2^{n-1}-k-1 ; x^{2}\right)+a\left(2^{n-1}-k ; x^{2}\right) \\
= & x\left(\left(x^{2}\right)^{2^{m}-k-1} a\left(k+1 ; x^{2}\right) a\left(2^{n-1-m}-1 ;\left(x^{2}\right)^{2^{m}}\right)+a\left(2^{m}-k-1 ; x^{2}\right)\right) \\
& \quad+\left(x^{2}\right)^{2^{m}-k} a\left(k ; x^{2}\right) a\left(2^{n-1-m}-1 ;\left(x^{2}\right)^{2^{m}}\right)+a\left(2^{m}-k ; x^{2}\right) \\
= & x^{2^{m+1}-(2 k+1)}\left[a\left(k+1 ; x^{2}\right)+x a\left(k ; x^{2}\right)\right] a\left(2^{n-(m+1)}-1 ; x^{2^{m+1}}\right) \\
& \quad+\left[x a\left(2^{m}-k-1 ; x^{2}\right)+a\left(2^{m}-k ; x^{2}\right)\right] \\
= & x^{2^{m+1}-(2 k+1)} a(2 k+1 ; x) a\left(2^{n-(m+1)}-1 ; x^{2^{m+1}}\right) \\
& +a\left(2^{m+1}-(2 k+1) ; x\right),
\end{aligned}
$$

where we have used (1.3) twice to simplify the terms in square brackets. If we replace $m+1$ by $m$ in both cases we have the result for $1 \leq m \leq n$, and $m=0$ is already known. This completes the induction.

3. The sequences $f_{n}(q)$ and $\bar{f}_{n}(q)$. As mentioned in the introduction, the maximum value of the Stern sequence $a(m)$ in the interval $2^{n-2} \leq m$ $\leq 2^{n-1}, n \geq 2$, is the $n$th Fibonacci number $F_{n}$ with the usual determination $F_{0}=0, F_{1}=1$, and

$$
F_{n+1}=F_{n}+F_{n-1} \quad(n \geq 1) .
$$

It was apparently first shown by Lehmer [17] that this maximum occurs at

$$
m=\frac{1}{3}\left(2^{n}-(-1)^{n}\right) \quad \text { and } \quad m=\frac{1}{3}\left(5 \cdot 2^{n-2}+(-1)^{n}\right) ;
$$

see also [18] or [25]. We will now use these values to define two subsequences of the Stern polynomials which will be the main objects of study in this paper. For this purpose we set

$$
\alpha_{n}:=\frac{1}{3}\left(2^{n}-(-1)^{n}\right) \quad(n \geq 0), \quad \beta_{n}:=\frac{1}{3}\left(5 \cdot 2^{n-2}+(-1)^{n}\right) \quad(n \geq 2) .
$$


The following properties are easy to verify (see also [25]):

$$
\begin{aligned}
\alpha_{n} & =2^{n-1}-2^{n-2}+\cdots+(-1)^{n-2} 2+(-1)^{n-1} \quad(n \geq 2), \\
\alpha_{n+1} & =2 \alpha_{n}+(-1)^{n}, \\
\alpha_{n+1} & =2^{n}-\alpha_{n}, \quad \alpha_{n-1}=2^{n-1}-\alpha_{n}, \\
\beta_{n+1} & =2 \beta_{n}-(-1)^{n}, \\
\beta_{n+1} & =2^{n}-\alpha_{n-1}, \quad \beta_{n+1}=2^{n-1}+\alpha_{n}, \\
\beta_{n} & =\alpha_{n+1}-2^{n-2} .
\end{aligned}
$$

The sequence $\left\{\alpha_{n}\right\}$ is sometimes called the Jacobsthal sequence; see [25, p. 186], or [24, A001045] for further properties and numerous references. The sequence $\left\{\beta_{n}\right\}$ can be found in [24, A048573]; the first few values of both are listed in Table 2 .

Table 2. $\alpha_{n}, \beta_{n}, 1 \leq n \leq 10$

\begin{tabular}{rrrrrrrrrrr}
\hline$n$ & 1 & 2 & 3 & 4 & 5 & 6 & 7 & 8 & 9 & 10 \\
\hline$\alpha_{n}$ & 1 & 1 & 3 & 5 & 11 & 21 & 43 & 85 & 171 & 341 \\
$\beta_{n}$ & & 2 & 3 & 7 & 13 & 27 & 53 & 107 & 213 & 427 \\
\hline
\end{tabular}

We now define the main objects of this section.

Definition 3.1. For each $n \geq 0$ we define

$$
f_{n}(q):=a\left(\alpha_{n} ; q\right),
$$

and for each $n \geq 2$,

$$
\bar{f}_{n}(q):=a\left(\beta_{n} ; q\right),
$$

so that in particular we have $f_{0}(q)=0$ and $f_{1}(q)=f_{2}(q)=\bar{f}_{2}(q)=1$ identically for all $q$.

The polynomials $f_{n}(q)$ and $\bar{f}_{n}(q)$ for the first few values of $n$ are listed in Table 3. The following important properties could be used as alternative definitions of these polynomials.

Table 3. $f_{n}(q)$ and $\bar{f}_{n}(q), 2 \leq n \leq 7$

\begin{tabular}{lll}
\hline$n$ & \multicolumn{1}{c}{$f_{n}(q)$} & \multicolumn{1}{c}{$\bar{f}_{n}(q)$} \\
\hline 2 & 1 & 1 \\
3 & $1+q$ & $1+q$ \\
4 & $1+q+q^{2}$ & $1+q+q^{3}$ \\
5 & $1+q+q^{3}+q^{4}+q^{5}$ & $1+q+q^{2}+q^{5}+q^{6}$ \\
6 & $1+q+q^{2}+q^{5}+q^{6}+q^{8}+q^{9}+q^{10}$ & $1+q+q^{3}+q^{4}+q^{5}+q^{11}+q^{12}+q^{13}$ \\
7 & $1+q+q^{3}+q^{4}+q^{5}+q^{11}+q^{12}$ & $1+q+q^{2}+q^{5}+q^{6}+q^{8}+q^{9}$ \\
& \multicolumn{1}{c}{$+q^{13}+q^{16}+q^{17}+q^{19}+q^{20}+q^{21}$} & $+q^{10}+q^{21}+q^{22}+q^{24}+q^{25}+q^{26}$ \\
\hline
\end{tabular}


Proposition 3.1. The polynomials $f_{n}(q)$ and $\bar{f}_{n}(q)$ satisfy

$$
\begin{aligned}
& f_{n+1}(q)=q^{\alpha_{n-1}} f_{n}(q)+f_{n-1}(q) \quad(n \geq 1), \\
& \bar{f}_{n+1}(q)=f_{n}(q)+q^{\alpha_{n}} f_{n-1}(q) \quad(n \geq 1), \\
& f_{n+1}(q)=\bar{f}_{n}(q)+q^{2^{n-2}} f_{n-1}(q) \quad(n \geq 2) .
\end{aligned}
$$

Proof. We use (2.2) with $m=n-1$ to obtain

$$
a\left(2^{n}-j ; q\right)=q^{2^{n-1}-j} a(j ; q)+a\left(2^{n-1}-j ; q\right) .
$$

If we take $j=\alpha_{n}$, then the two identities in (3.5) together with the definition (3.9) immediately give (3.11).

Next we use (3.14) with $j=\alpha_{n-1}$. Then we have $2^{n}-j=\beta_{n+1}$ by (3.7), and $2^{n-1}-j=\alpha_{n}$ by (3.5). So (3.14) gives

$$
a\left(\beta_{n+1} ; q\right)=q^{\alpha_{n}} a\left(\alpha_{n-1} ; q\right)+a\left(\alpha_{n} ; q\right),
$$

which is the same as (3.12), by (3.10) and (3.9).

For the third identity we use (2.4) with $m=n-2$ and $j=\alpha_{n-1}$. Then $2^{m+1}-j=\alpha_{n}$ by (3.5), and $2^{m}+j=\beta_{n}$ by (3.7). So (2.4) gives, again with (3.10) and (3.9),

$$
q^{\alpha_{n-1}} f_{n}(q)=\left(q^{2^{n-2}}-1\right) f_{n-1}(q)+\bar{f}_{n}(q) .
$$

Finally, if we add (3.11) to this identity, we readily obtain (3.13).

If we set $q=1$ and compare the identities (3.11) and (3.12) with (3.1), we immediately see that

$$
f_{n}(1)=\bar{f}_{n}(1)=F_{n} .
$$

This, of course, is not surprising, given the way we defined the sequences $f_{n}(q)$ and $\bar{f}_{n}(q)$. It can also be shown that $(1+q) \mid f_{n}(q)+\bar{f}_{n}(q)$. In fact,

$$
f_{n}(-1)=-\bar{f}_{n}(-1)=(-1)^{n} F_{n-3} .
$$

At this point we remark that various $q$-analogues of Fibonacci numbers have been known for a long time (see, e.g., [7], [8]) and are still of current interest (see, e.g., [9]). Usually, these analogues are such that they relate to the $q$-binomial coefficients much as the Fibonacci numbers relate to the binomial coefficients. There is also a vast literature on Fibonacci (and Lucas) polynomials; they are closely related to the Chebyshev polynomials of both kinds.

The identities in Proposition 3.1 lead to another linear recurrence relation that will be needed in the proof of Proposition 5.3.

Corollary 3.1. For all $n \geq 2$ we have

$$
f_{n+2}(q)=\left(1+q^{2^{n-2}}+q^{2^{n-1}}\right) f_{n}(q)-q^{2^{n-2}} f_{n-2}(q) .
$$


Proof. We replace $n$ by $n+1$ in (3.13) and combine it with (3.12), to get

$$
f_{n+2}(q)=\left(1+q^{2^{n-1}}\right) f_{n}(q)+q^{\alpha_{n}} f_{n-1}(q) .
$$

Next we replace $n$ by $n-1$ in (3.11) and multiply both sides by $q^{2^{n-2}}$. Noting that the two identities in (3.5) give $\alpha_{n-2}+2^{n-2}=\alpha_{n}$, we obtain

$$
q^{2^{n-2}} f_{n}(q)=q^{\alpha_{n}} f_{n-1}(q)+q^{2^{n-2}} f_{n-2}(q) .
$$

This, combined with (3.17), gives (3.16).

It can be seen from Tables 2 and 3 that the degrees of the polynomials are closely related to the integers $\alpha_{n-1}$, resp. $\beta_{n-1}$. In fact, we have

Proposition 3.2. The coefficients of $f_{n}(q)$ and $\bar{f}_{n}(q)$ are 0 or 1 , and for $k \geq 1$ we have

$$
\begin{aligned}
\operatorname{deg} f_{2 k}(q) & =\alpha_{2 k-1}-1, & & \operatorname{deg} f_{2 k+1}(q)=\alpha_{2 k}, \\
\operatorname{deg} \bar{f}_{2 k}(q) & =\beta_{2 k-1} \quad(k \geq 2), & & \operatorname{deg} \bar{f}_{2 k+1}(q)=\beta_{2 k}-1 .
\end{aligned}
$$

Proof. In Proposition 2.1 of [13] it was shown that the Stern polynomials have only 0 or 1 as coefficients; this carries over to the $f_{n}(q)$ and $\bar{f}_{n}(q)$. It was also shown there that for $n \geq 0$,

$$
\operatorname{deg} a(2 n+1 ; q)=n .
$$

Now by (3.4) we have

$$
\alpha_{2 k}=2\left(\alpha_{2 k-1}-1\right)+1, \quad \alpha_{2 k+1}=2 \alpha_{2 k}+1,
$$

which immediately leads to (3.18). The proof of (3.19) is completely analogous, using (3.20) and this time (3.6).

Alternatively, Proposition 3.2 could be proved by induction, using the recurrence relations in Proposition 3.1.

In addition to (3.11)-(3.13), the following recurrence relations are also analogues to (3.1).

Proposition 3.3. For any $k \geq 1$ we have

$$
\begin{aligned}
f_{2 k}(q) & =f_{2 k-1}\left(q^{2}\right)+q f_{2 k-2}\left(q^{4}\right), \\
f_{2 k+1}(q) & =q f_{2 k}\left(q^{2}\right)+f_{2 k-1}\left(q^{4}\right),
\end{aligned}
$$

and for $k \geq 2$,

$$
\begin{aligned}
\bar{f}_{2 k}(q) & =q \bar{f}_{2 k-1}\left(q^{2}\right)+\bar{f}_{2 k-2}\left(q^{4}\right), \\
\bar{f}_{2 k+1}(q) & =\bar{f}_{2 k}\left(q^{2}\right)+q \bar{f}_{2 k-1}\left(q^{4}\right) .
\end{aligned}
$$


Proof. To obtain (3.21), we use (3.4) and then (1.3) with $n=\alpha_{2 k-1}-1$, followed by (1.2):

$$
\begin{aligned}
f_{2 k}(q) & =a\left(\alpha_{2 k} ; q\right)=a\left(2 \alpha_{2 k-1}-1 ; q\right)=a\left(\alpha_{2 k-1} ; q^{2}\right)+q a\left(\alpha_{2 k-1}-1 ; q^{2}\right) \\
& =a\left(\alpha_{2 k-1} ; q^{2}\right)+q a\left(2 \alpha_{2 k-2} ; q^{2}\right)=a\left(\alpha_{2 k-1} ; q^{2}\right)+q a\left(\alpha_{2 k-2} ; q^{4}\right),
\end{aligned}
$$

and this last expression is clearly the right-hand side of (3.21). The proofs of (3.22)-(3.24) are analogous.

An important consequence of (3.11) is the fact that for each $n$ the polynomials $f_{n+1}(q)$ and $f_{n-1}(q)$ agree up to and including the $\alpha_{n-1}$ th power of $q$. The following result shows that the polynomials $\bar{f}_{n}(q)$ have a similar property.

Proposition 3.4. For all $n \geq 3$ we have

$$
\bar{f}_{n+1}(q)=\bar{f}_{n-1}(q)+q^{2^{n-3}}\left(q^{\beta_{n-1}} f_{n-1}(q)+f_{n-2}(q)\right) .
$$

Proof. Substituting (3.13) with $n$ replaced by $n-1$ into (3.12), we get

$$
\bar{f}_{n+1}(q)=\bar{f}_{n-1}(q)+q^{2^{n-3}}\left(q^{\alpha_{n}-2^{n-3}} f_{n-1}(q)+f_{n-2}(q)\right) .
$$

The result now follows from (3.8).

4. Nonlinear relations. One of the best known identities for the Fibonacci numbers, apart from the basic recurrence (3.1), is (for $n \geq 1$ )

$$
F_{n-1} F_{n+1}-F_{n}^{2}=(-1)^{n} .
$$

In this section we derive several analogues of this identity.

Proposition 4.1. For all $k \geq 1$ we have

$$
\begin{aligned}
f_{2 k+1}(q) f_{2 k-1}\left(q^{2}\right)-q f_{2 k}(q) f_{2 k}\left(q^{2}\right) & =1, \\
f_{2 k+1}(q) f_{2 k+1}\left(q^{2}\right)-q f_{2 k+2}(q) f_{2 k}\left(q^{2}\right) & =1 .
\end{aligned}
$$

Proof. It is easy to check that both identities hold for $k=1$. Suppose now that they hold up to some $k-1$. We use (3.11) to rewrite the left-hand side of (4.2) as

$$
\begin{array}{r}
\left(q^{\alpha_{2 k-1}} f_{2 k}(q)+f_{2 k-1}(q)\right) f_{2 k-1}\left(q^{2}\right)-q f_{2 k}(q)\left(q^{2 \alpha_{2 k-2}} f_{2 k-1}\left(q^{2}\right)+f_{2 k-2}\left(q^{2}\right)\right) \\
=f_{2 k-1}(q) f_{2 k-1}\left(q^{2}\right)-q f_{2 k}(q) f_{2 k-2}\left(q^{2}\right)=1
\end{array}
$$

by (3.4), and by (4.3) with $k$ replaced by $k-1$. Similarly we rewrite the left-hand side of (4.3), this time using (4.2) together with (3.11) and (3.4):

$$
\begin{aligned}
f_{2 k+1}(q)\left(q^{2 \alpha_{2 k-1}} f_{2 k}\left(q^{2}\right)+f_{2 k-1}\left(q^{2}\right)\right)-q\left(q^{\alpha_{2 k}} f_{2 k+1}(q)+f_{2 k}(q)\right) f_{2 k}\left(q^{2}\right) \\
=f_{2 k+1}(q) f_{2 k-1}\left(q^{2}\right)-q f_{2 k}(q) f_{2 k}\left(q^{2}\right)=1
\end{aligned}
$$

by (4.2), where we have used (3.4) again. This completes the proof by induction.

We now prove another pair of analogues to (3.1). 
Proposition 4.2. For $k \geq 2$ we have

$$
f_{2 k+1}(q) \bar{f}_{2 k-1}\left(q^{2}\right)-\bar{f}_{2 k}(q) f_{2 k}\left(q^{2}\right)=q^{\beta_{2 k}-1},
$$

and for $k \geq 1$,

$$
\bar{f}_{2 k+2}(q) f_{2 k}\left(q^{2}\right)-f_{2 k+1}(q) \bar{f}_{2 k+1}\left(q^{2}\right)=-q^{\alpha_{2 k+1}-1} .
$$

Proof. We begin with (4.5). Using (3.12) we get

$$
\begin{aligned}
\bar{f}_{2 k+2}(q) & f_{2 k}\left(q^{2}\right)-f_{2 k+1}(q) \bar{f}_{2 k+1}\left(q^{2}\right) \\
= & \left(f_{2 k+1}(q)+q^{\alpha_{2 k+1}} f_{2 k}(q)\right) f_{2 k}\left(q^{2}\right) \\
& -f_{2 k+1}(q)\left(f_{2 k}\left(q^{2}\right)+q^{2 \alpha_{2 k}} f_{2 k-1}\left(q^{2}\right)\right) \\
= & q^{\alpha_{2 k+1}-1}\left(q f_{2 k}(q) f_{2 k}\left(q^{2}\right)-f_{2 k+1}(q) f_{2 k-1}\left(q^{2}\right)\right)=-q^{\alpha_{2 k+1}-1},
\end{aligned}
$$

where we have used (3.4) and (4.2). Next, (3.13) leads to

$$
\begin{aligned}
& f_{2 k+1}(q) \bar{f}_{2 k-1}\left(q^{2}\right)-\bar{f}_{2 k}(q) f_{2 k}\left(q^{2}\right) \\
& =\left(\bar{f}_{2 k}(q)+q^{2^{2 k-2}} f_{2 k-1}(q)\right) \bar{f}_{2 k-1}\left(q^{2}\right)-\bar{f}_{2 k}(q)\left(\bar{f}_{2 k-1}\left(q^{2}\right)+q^{2 \cdot 2^{2 k-3}} f_{2 k-2}\left(q^{2}\right)\right) \\
& =q^{2^{2 k-2}}\left(f_{2 k-1}(q) \bar{f}_{2 k-1}\left(q^{2}\right)-\bar{f}_{2 k}(q) f_{2 k-2}\left(q^{2}\right)\right) \\
& =-q^{2^{2 k-2}} q^{\alpha_{2 k-1}-1}=-q^{\beta_{2 k}-1},
\end{aligned}
$$

where the last two equalities follow from (4.5) and (3.7), respectively. This proves the identity (4.4).

REMARK. The sequence of exponents on the right-hand sides of (4.4) and (4.5) is interesting in its own right. We set $\gamma_{n}=\beta_{n}-1$ when $n$ is even, and $\gamma_{n}=\alpha_{n}-1$ when $n$ is odd. In Table 4 we list the first few values.

Table 4. $\gamma_{n}, 1 \leq n \leq 14$

\begin{tabular}{ccccccccccccccc}
\hline$n$ & 1 & 2 & 3 & 4 & 5 & 6 & 7 & 8 & 9 & 10 & 11 & 12 & 13 & 14 \\
\hline$\gamma_{n}$ & 0 & 1 & 2 & 6 & 10 & 26 & 42 & 106 & 170 & 426 & 682 & 1706 & 2730 & 6826 \\
\hline
\end{tabular}

The numbers $\gamma_{n}$ are known to have a simple combinatorial interpretation: For a given $n \geq 1, \gamma_{n}$ is the number of 132- and 213-avoiding derangements of the set $\{1, \ldots, n\}$; see [24, A061547]. This last reference also gives the explicit formula

$$
\gamma_{n}=\frac{3}{8} \cdot 2^{n}+\frac{1}{24} \cdot(-2)^{n}-\frac{2}{3},
$$

and the recurrence relation

$$
\gamma_{1}=0, \quad \gamma_{2}=1, \quad \gamma_{n}=4 \gamma_{n-2}+2 .
$$

Both properties are easy to derive from the definition (3.2). 
5. Two limit functions. At the end of Section 3 we already remarked that, by (3.11), for each $n$ the polynomials $f_{n+1}(q)$ and $f_{n-1}(q)$ agree up to and including the $\alpha_{n-1}$ th power of $q$. Similarly, the identity (3.25) shows that $\bar{f}_{n+1}(q)$ and $\bar{f}_{n-1}(q)$ agree up to and including the $2^{n-3}$ th power of $q$. Finally, by (3.12) and (3.13), similar agreements exist between $\bar{f}_{n+1}(q)$ and $f_{n}(q)$, and between $f_{n+1}(q)$ and $\bar{f}_{n}(q)$, respectively. This means that the following two functions are well-defined.

Definition 5.1. For complex $q$ with $|q|<1$ we define

$$
\begin{aligned}
F(q) & :=\lim _{n \rightarrow \infty} f_{2 n}(q)=\lim _{n \rightarrow \infty} \bar{f}_{2 n+1}(q) \\
& =1+q+q^{2}+q^{5}+q^{6}+q^{8}+q^{9}+q^{10}+q^{21}+q^{22}+q^{24}+\cdots, \\
G(q) & :=\lim _{n \rightarrow \infty} f_{2 n+1}(q)=\lim _{n \rightarrow \infty} \bar{f}_{2 n}(q) \\
& =1+q+q^{3}+q^{4}+q^{5}+q^{11}+q^{12}+q^{13}+q^{16}+q^{17}+q^{19}+\cdots .
\end{aligned}
$$

It is clear that both these functions are analytic for $|q|<1$. Also, from (3.21) or (3.24), and from (3.22) or (3.23) they inherit the relationships

$$
\begin{aligned}
& F(q)=G\left(q^{2}\right)+q F\left(q^{4}\right), \\
& G(q)=q F\left(q^{2}\right)+G\left(q^{4}\right),
\end{aligned}
$$

respectively. The sequences of integers appearing in the exponents here are not found in [24] at the time of writing. Nonetheless, they are simple examples of so-called "self-generating sequences". In fact, if $\Phi$ and $\Gamma$ are minimal sets of positive integers such that $1 \in \Phi \cap \Gamma$ and

$$
\Phi \supseteq(4 \Phi+1) \cup 2 \Gamma, \quad \Gamma \supseteq(2 \Phi+1) \cup 4 \Gamma,
$$

then $\Phi$ is the set of exponents of $F$ and $\Gamma$ is the set of exponents of $G$. For properties of sequences defined in a similar manner (though not the particular ones occurring here) see [5], [14], or [15].

We now show that the linked functional equations (5.3) and (5.4) lead to functional equations for $F$ alone, and for $G$ alone.

Proposition 5.1. For $|q|<1$ we have

$$
\begin{aligned}
F(q) & =\left(1+q+q^{2}\right) F\left(q^{4}\right)-q^{4} F\left(q^{16}\right), \\
q G(q) & =\left(1+q+q^{2}\right) G\left(q^{4}\right)-G\left(q^{16}\right) .
\end{aligned}
$$

Proof. Replacing $q$ by $q^{2}$ in (5.3) and eliminating $G\left(q^{4}\right)$ with the help of (5.4), we obtain

$$
G(q)=(1+q) F\left(q^{2}\right)-q^{2} F\left(q^{8}\right) .
$$

Then we replace $q$ by $q^{2}$ in this identity and substitute it into (5.3). This gives (5.5). The identity (5.6) is obtained with similar manipulations, using again (5.3) and (5.4). 
The two functions $F$ and $G$ also satisfy nonlinear functional equations:

Proposition 5.2. For $|q|<1$ we have

$$
\begin{aligned}
& G(q) G\left(q^{2}\right)-q F(q) F\left(q^{2}\right)=1, \\
& F(q) G\left(q^{4}\right)-q G(q) F\left(q^{4}\right)=1 .
\end{aligned}
$$

Proof. The identity (5.7) follows directly from (4.2) or (4.3) by taking limits. If we substitute $G\left(q^{2}\right)$ and $q F\left(q^{2}\right)$ with the help of (5.3) and (5.4), respectively, we easily obtain (5.8).

We can actually go further: Multiply both sides of (5.8) by $1+q+q^{2}$ and use (5.5) and (5.6) to eliminate $F\left(q^{4}\right)$ and $G\left(q^{4}\right)$. Then after some simplification we get

$$
F(q) G\left(q^{16}\right)-q^{5} G(q) F\left(q^{16}\right)=1+q+q^{2} .
$$

It turns out that (5.8) and (5.9) are special cases of the following infinite class of identities.

Proposition 5.3. For all $n \geq 1$ and $|q|<1$ we have

$$
F(q) G\left(q^{2^{2 n}}\right)-q^{\alpha_{2 n}} G(q) F\left(q^{2^{2 n}}\right)=f_{2 n}(q) .
$$

Proof. We proceed by induction on $n$. For $n=1$ and $n=2$ the identity reduces to (5.8) and (5.9), respectively; see also Tables 2 and 3. (Note that (5.10) is trivially true for $n=0$ since $f_{0}(q)=0$.) Suppose now that $(5.10)$ holds up to a certain fixed $n \geq 1$. We multiply both sides of (5.10) by

$$
Q_{n}:=1+q^{2^{2 n-2}}+q^{2^{2 n-1}} .
$$

Using (5.5) and (5.6), with $q$ replaced by $q^{2^{2 n-2}}$, we then get

$$
\begin{aligned}
Q_{n} f_{2 n}(q)= & F(q)\left[q^{2^{2 n-2}} G\left(q^{2^{2 n-2}}\right)+G\left(q^{2^{2 n+2}}\right)\right] \\
& -q^{\alpha_{2 n}} G(q)\left[F\left(q^{2^{2 n-2}}\right)+q^{2^{2 n}} F\left(q^{2^{2 n+2}}\right)\right] \\
= & q^{2^{2 n-2}}\left[F(q) G\left(q^{2^{2 n-2}}\right)-q^{\alpha_{2 n}-2^{2 n-2}} G(q) F\left(q^{2^{2 n-2}}\right)\right] \\
& +F(q) G\left(q^{2^{2 n+2}}\right)-q^{\alpha_{2 n}+2^{2 n}} G(q) F\left(q^{2^{2 n+2}}\right) .
\end{aligned}
$$

Now by the two identities in (3.5) we have $\alpha_{2 n}-2^{2 n-2}=\alpha_{2 n-2}$, and therefore by the induction hypothesis (5.10) the last expression in square brackets is simply $f_{2 n-2}(q)$. Then we use the fact that by Corollary 3.1 we have

$$
Q_{n} f_{2 n}(q)-q^{2^{2 n-2}} f_{2 n-2}(q)=f_{2 n+2}(q),
$$

which means that we have obtained (5.10) with $n+1$ for $n$; it only remains to remark that $\alpha_{2 n}+2^{2 n}=\alpha_{2 n+2}$, again by (3.5). This completes the proof by induction.

Functional equations similar to (5.5) and (5.6) were studied in [20] in connection with a "paper-folding" sequence. The main theorem in [20] does 
not apply directly to (5.5) and (5.6), but using a result of Nishioka [21], Adamczewski [1] was able to prove

Proposition 5.4 (Adamczewski). The functions $F$ and $G$ take transcendental values at every algebraic number $q, 0<|q|<1$.

Independently, M. Coons [10] recently proved that $F$ and $G$ are transcendental functions, along with results (including transcendence at algebraic numbers) related to functions that occur in [13].

6. Continued fractions. There is a well-known and close relationship between Fibonacci numbers and finite simple continued fractions denoted by

$$
\left\langle a_{0}, a_{1}, \ldots, a_{n}\right\rangle=a_{0}+\frac{1}{a_{1}+\frac{1}{\ddots \cdot a_{n-1}+\frac{1}{a_{n}}}}=\frac{h_{n}}{k_{n}},
$$

where the terms $a_{j}$ (also called partial quotients) are usually positive integers. The convergents $h_{n} / k_{n}$ are related to the $a_{j}$ by the recurrence relations

$$
\begin{aligned}
& h_{j}=a_{j} h_{j-1}+h_{j-2}, \\
& k_{j}=a_{j} k_{j-1}+k_{j-2},
\end{aligned}
$$

with $h_{-2}=0, h_{-1}=1$ and $k_{-2}=1, k_{-1}=0$; see, e.g., [22, Ch. 7] for further details. The simplest of the finite simple continued fractions are

$$
\frac{F_{n+1}}{F_{n}}=\langle 1,1, \ldots, 1\rangle \quad(n \text { terms }),
$$

with the corresponding infinite continued fraction

$$
\frac{\sqrt{5}+1}{2}=\langle 1,1,1, \ldots\rangle=\lim _{n \rightarrow \infty} \frac{h_{n}}{k_{n}} .
$$

In this section we shall derive analogues to (6.4) and (6.5) involving the polynomials $f_{n}(q)$ and the functions $F(q), G(q)$. We begin with the following finite continued fraction.

Proposition 6.1. For $n \geq 0$ and $q \neq 0$ we have

$$
\left\langle q^{2^{n}}, q^{2^{n-1}}, \ldots, q^{4}, q^{2}, q\right\rangle=q^{(-1)^{n}} \frac{f_{n+2}\left(q^{3}\right)}{f_{n+1}\left(q^{3}\right)} .
$$

Proof. We use (3.11) with $q$ replaced by $q^{3}$ and $n$ replaced by $n+1$, and we note that $3 \alpha_{n}=2^{n}-(-1)^{n}$ by (3.2). Then we have

$$
q^{(-1)^{n+2}} \frac{f_{n+2}\left(q^{3}\right)}{f_{n+1}\left(q^{3}\right)}=q^{2^{n}}+\left(q^{(-1)^{n+1}} \frac{f_{n+1}\left(q^{3}\right)}{f_{n}\left(q^{3}\right)}\right)^{-1} .
$$


Iterating this and using the fact that the last term will be

$$
q^{(-1)^{2}} \frac{f_{2}\left(q^{3}\right)}{f_{1}\left(q^{3}\right)}=q
$$

(see Table 3), we immediately get (6.6).

The following interesting result will be useful for what follows. It can be found, e.g., in [2].

Lemma 6.1 (The mirror formula). Let $a_{0}, a_{1}, \ldots$ be positive integers. Let

$$
\frac{p_{n}}{q_{n}}=\left\langle a_{0}, a_{1}, \ldots, a_{n}\right\rangle .
$$

Then

$$
\frac{q_{n}}{q_{n-1}}=\left\langle a_{n}, a_{n-1}, \ldots, a_{1}\right\rangle .
$$

We use this formula to obtain an evaluation for the following finite continued fraction.

Proposition 6.2. For $n \geq 0$ and $q \neq 0$ we have

$$
\left\langle q, q^{2}, q^{4}, \ldots, q^{2^{n}}\right\rangle=q^{\left(3(-1)^{n}-1\right) / 2} \frac{f_{n+2}\left(q^{3}\right)}{f_{n+1}\left(q^{6}\right)} .
$$

Proof. We first suppose that $n$ is even, and we use (6.6) with $n$ replaced by $n+1$. In the notation of (6.7) we then have

$$
\frac{p_{n}}{q_{n}}=\left\langle q^{2^{n+1}}, q^{2^{n}}, \ldots, q^{4}, q^{2}, q\right\rangle=\frac{f_{n+3}\left(q^{3}\right)}{q f_{n+2}\left(q^{3}\right)} .
$$

Similarly, using (6.6) with $q$ replaced by $q^{2}$, we get

$$
\begin{aligned}
\frac{p_{n-1}}{q_{n-1}} & =\left\langle q^{2^{n+1}}, q^{2^{n}}, \ldots, q^{4}, q^{2}\right\rangle \\
& =\left\langle\left(q^{2}\right)^{2^{n}},\left(q^{2}\right)^{2^{n-1}}, \ldots,\left(q^{2}\right)^{2},\left(q^{2}\right)^{1}\right\rangle=\frac{q^{2} f_{n+2}\left(q^{6}\right)}{f_{n+1}\left(q^{6}\right)} .
\end{aligned}
$$

Hence by the mirror formula the continued fraction in (6.9) is just $q_{n} / q_{n-1}$, which is the right-hand side of (6.9) for $n$ even. The case when $n$ is odd is completely analogous.

When $q$ is a positive integer, then by the theory of simple continued fractions the limit

$$
c_{q}:=\lim _{n \rightarrow \infty}\left\langle q, q^{2}, q^{4}, \ldots, q^{2^{n}}\right\rangle=\left\langle q, q^{2}, q^{4}, \ldots\right\rangle
$$

exists and lies always between two successive convergents. This makes it easy to compute the values to any accuracy, using, for instance, the identity (3.11) (which is equivalent to (6.2) and (6.3)). Convergence will obviously be very 
fast when $q \geq 2$, and already $n=2$ in (6.9) gives a very good approximation for $q \geq 2$, so that

$$
c_{q} \simeq q \frac{1+q^{-3}+q^{-6}}{1+q^{-6}},
$$

with an error that is easily quantifiable.

While $c_{1}=(1+\sqrt{5}) / 2$ is obviously algebraic, we shall see in the next section that $c_{q}$ is transcendental for any integer $q \geq 2$.

When $|q|<1$, the situation is drastically different. The theory of simple continued fractions no longer applies, and in particular the infinite analogue of the left-hand side of (6.9), i.e., the limit as $n \rightarrow \infty$, may no longer exist. In fact, by the Stern-Stolz theorem (see, e.g., [19, p. 94]) the limit cannot exist for $|q|<1$. However, we can use the functions $F$ and $G$ defined in (5.1) and (5.2) to obtain the following limit result, which is an obvious consequence of $(6.9)$.

Proposition 6.3. Whenever $0<|q|<1$, we have

$$
\begin{aligned}
& \lim _{\substack{n \rightarrow \infty \\
n \text { even }}}\left\langle q, q^{2}, q^{4}, \ldots, q^{2^{n}}\right\rangle=\frac{q F\left(q^{3}\right)}{G\left(q^{6}\right)}, \\
& \lim _{\substack{n \rightarrow \infty \\
n \text { odd }}}\left\langle q, q^{2}, q^{4}, \ldots, q^{2^{n}}\right\rangle=\frac{G\left(q^{3}\right)}{q^{2} F\left(q^{6}\right)} .
\end{aligned}
$$

Thus, while the limit as such does not exist, there are two partial limits.

REMARKS.

1. A general discussion of divergent continued fractions with multiple limits can be found in [6].

2. Different classes of interesting doubly exponential continued fractions were introduced and studied in [4] and [12].

As a consequence of Proposition 6.3 we obtain the following result.

Proposition 6.4. For any $|q|<1$, we have $F(q) \neq 0$ and $G(q) \neq 0$.

Proof. Part of the theorem of Stern and Stolz is the statement that both the limits in (6.11) and (6.12) are finite. Hence, if we had $G\left(q^{6}\right)=0$ for some $q$ with $|q|<1$, then also $F\left(q^{3}\right)=0$. But then, by (5.3) and (5.4), we have $F\left(q^{12}\right)=0$ and $G\left(q^{24}\right)=0$, respectively. Iterating this, we find that

$$
F\left(q^{3 \cdot 4^{k}}\right)=G\left(q^{6 \cdot 4^{k}}\right)=0
$$

for all $k \geq 0$. This contradicts the fact that the analytic and hence continuous functions $F$ and $G$ take the value 1 at $q=0$.

Similarly, if $F\left(q^{6}\right)=0$ then by (6.12) we also have $G\left(q^{3}\right)=0$, and (5.4) and (5.3) imply $G\left(q^{12}\right)=F\left(q^{24}\right)=0$. Just as before, we iterate this and obtain a contradiction. This completes the proof. 


\section{Further remarks}

1. While the continued fraction $c_{1}=\langle 1,1,1, \ldots\rangle$ is clearly algebraic, we are going to prove

Proposition 7.1. For any integer $q \geq 2$ the infinite continued fraction

$$
c_{q}=\left\langle q, q^{2}, q^{4}, \ldots, q^{2^{n}}, \ldots\right\rangle
$$

is transcendental.

Proof. We could use Roth's theorem, but it will be easier to apply a later theorem of Davenport and Roth [11]. It states that, given an algebraic number $\alpha$ whose continued fraction expansion has $p_{n} / q_{n}$ as its $n$th convergent, we have

$$
\log \log q_{n}<c(\alpha) \frac{n}{\sqrt{\log n}},
$$

where $c(\alpha)$ is independent of $n$. Now it follows from (7.1) and the theory of continued fractions that $q_{n} \geq q^{2^{n}}$, and obviously (7.2) cannot be satisfied; hence $c_{q}$ is transcendental for $q \geq 2$.

For a recent improvement of the theorem of Davenport and Roth, see [3].

2. For the case $|q|<1$, Adamczewski [1] proved the following result.

Proposition 7.2 (Adamczewski). Let $q$ be an algebraic number with $0<|q|<1$. Then both the limits in (6.11) and (6.12) are transcendental.

Acknowledgments. We would like to thank Boris Adamczewski for a careful reading of this paper, and for suggesting the current substantially shorter proof of Proposition 7.1.

Research supported in part by the Natural Sciences and Engineering Research Council of Canada.

\section{References}

[1] B. Adamczewski, Non-converging continued fractions related to the Stern diatomic sequence, Acta Arith., to appear.

[2] B. Adamczewski and J.-P. Allouche, Reversals and palindromes in continued fractions, Theoret. Comput. Sci. 380 (2007), 220-237.

[3] B. Adamczewski and Y. Bugeaud, On the Maillet-Baker continued fractions, J. Reine Angew. Math. 606 (2007), 105-121.

[4] W. W. Adams and J. L. Davison, A remarkable class of continued fractions, Proc. Amer. Math. Soc. 65 (1977), 194-198.

[5] J.-P. Allouche, J. Shallit, and G. Skordev, Self-generating sets, integers with missing blocks, and substitutions, Discrete Math. 292 (2005), 1-15.

[6] D. Bowman and J. Mc Laughlin, Continued fractions with multiple limits, Adv. Math. 210 (2007), 578-606. 
[7] L. Carlitz, Fibonacci notes. III. q-Fibonacci numbers, Fibonacci Quart. 12 (1974), $317-322$.

[8] L. Carlitz, Fibonacci notes. IV. q-Fibonacci polynomials, ibid. 13 (1975), 97-102.

[9] J. Cigler, A new class of q-Fibonacci polynomials, Electron. J. Combin. 10 (2003), Research Paper 19, 15 pp.

[10] M. Coons, The transcendence of series related to Stern's diatomic sequence, Int. J. Number Theory, to appear.

[11] H. Davenport and K. F. Roth, Rational approximations to algebraic numbers, Mathematika 2 (1955), 160-167.

[12] J. L. Davison, A series and its associated continued fraction, Proc. Amer. Math. Soc. 63 (1977), 29-32.

[13] K. Dilcher and K. B. Stolarsky, A polynomial analogue to the Stern sequence, Int. J. Number Theory 3 (2007), 85-103.

[14] D. Garth and A. Gouge, Affinely self-generating sets and morphisms, J. Integer Seq. 10 (2007), art. 07.1.5.

[15] C. Kimberling, Affinely recursive sets and ordering of languages, Discrete Math. 274 (2004), 147-159.

[16] S. Klavžar, U. Milutinović, and C. Petr, Stern polynomials, Adv. Appl. Math. 39 (2007), 86-95.

[17] D. H. Lehmer, On Stern's diatomic series, Amer. Math. Monthly 36 (1929), 59-67.

[18] D. A. Lind, An extension of Stern's diatomic series, Duke Math. J. 36 (1969), 55-60.

[19] L. Lorentzen and H. Waadeland, Continued Fractions with Applications, NorthHolland, Amsterdam, 1992.

[20] J. H. Loxton and A. J. van der Poorten, Arithmetic properties of the solutions of a class of functional equations, J. Reine Angew. Math. 330 (1982), 159-172.

[21] K. Nishioka, Mahler Functions and Transcendence, Lecture Notes in Math. 1631, Springer, Berlin, 1996.

[22] I. Niven, H. S. Zuckerman, and H. L. Montgomery, An Introduction to the Theory of Numbers, 5th ed., Wiley, New York, 1991.

[23] B. Reznick, Some binary partition functions, in: Analytic Number Theory, B. C. Berndt et al. (eds.), Birkhäuser, Boston, 1990, 451-477.

[24] N. J. A. Sloane, On-Line Encyclopedia of Integer Sequences, http://www.research. att.com $/ \sim$ njas/sequences/.

[25] I. Urbiha, Some properties of a function studied by de Rham, Carlitz and Dijkstra and its relation to the (Eisenstein-)Stern's diatomic sequence, Math. Comm. 6 (2001), 181-198.

Department of Mathematics and Statistics

Dalhousie University

Halifax, Nova Scotia, B3H 3J5, Canada

E-mail: dilcher@mathstat.dal.ca
Department of Mathematics University of Illinois 1409 West Green Street Urbana, IL 61801, U.S.A. E-mail: stolarsk@math.uiuc.edu

Received on 4.10.2008

and in revised form on 24.2.2009 